\title{
Excellent local control and tolerance profile after stereotactic body radiotherapy of advanced hepatocellular carcinoma
}

\author{
Eleni Gkika ${ }^{1 *}$, Michael Schultheiss ${ }^{2}$, Dominik Bettinger ${ }^{2,9}$, Lars Maruschke ${ }^{3}$, Hannes Philipp Neeff ${ }^{4}$, \\ Michaela Schulenburg ${ }^{5}$, Sonja Adebahr ${ }^{1,6}$, Simon Kirste ${ }^{1}$, Ursula Nestle ${ }^{1,6}$, Robert Thimme 2 , \\ Anca-Ligia Grosu ${ }^{1,6,7,8}$ and Thomas Baptist Brunner ${ }^{1,6,7,8}$
}

\begin{abstract}
Background: To evaluate the efficacy and toxicity of stereotactic body radiotherapy (SBRT) in the treatment of advanced hepatocellular carcinoma (HCC).

Material and Methods: Patients with large HCCs (median diameter $7 \mathrm{~cm}$, IQR 5-10 cm) with a Child-Turcotte-Pugh (CTP) score A (60\%) or B (40\%) and Barcelona-Clinic Liver Cancer (BCLC) classification stage B or C were treated with 3 to 12 fractions to allow personalized treatment according to the size of the lesions and the proximity of the lesions to the organs at risk aiming to give high biologically equivalent doses assuming an $\alpha / \beta$ ratio of 10 Gy for HCC. Primary end points were in-field local control and toxicity assessment.

Results: Forty seven patients with 64 lesions were treated with SBRT (median 45 Gy in 3-12 fractions) with a median follow up for patients alive of 19 months. The median biological effective dose was 76 Gy (IQR 62-86 Gy). Tumor vascular thrombosis was present in 28\% and an underlying liver disease in $87 \%$ (hepatitis B or C in 21\%, alcohol related in 51\%, nonalcoholic steatohepatitis in 13\% of the patients, primary biliary cirrhosis 2\%). Eighty three percent received prior and in most cases multiple therapies. Local control at 1 year was $77 \%$. The median overall survival from the start of SBRT was 9 months (95\% Cl 7.7-10.3). Gastrointestinal toxicities grade $\geq 2$ were observed in 3 (6.4\%) patients. An increase in CTP score without disease progression was observed in 5 patients, of whom one patient developed a radiation induced liver disease. One patient died due to liver failure 4 months after treatment.
\end{abstract}

Conclusion: SBRT is an effective local ablative therapy which leads to high local control rates with moderate toxicity for selected patients with large tumors.

Keywords: Stereotactic body radiotherapy, SBRT, Hepatocellular carcinoma, HCC, SIP

\section{Background}

Liver cancer is the seventh most common cancer worldwide, with more than 782,000 new cases diagnosed in 2012 (6\% of the total) and the second most common cause of cancer-related deaths worldwide after lung cancer, with more than 745,000 deaths annually [1].

Surgery is the mainstay of $\mathrm{HCC}$ treatment with a 5 year overall survival of $50 \%$ [2], yet only $15 \%$ of the newly

\footnotetext{
* Correspondence: eleni.gkika@uniklinik-freiburg.de

'Department of Radiation Oncology, University Medical Center, Freiburg, Germany

Full list of author information is available at the end of the article
}

diagnosed HCC-patients are eligible for surgery [3], transplantation or radiofrequency ablation (RFA). These treatment options are limited due to impaired liver function including portal hypertension, advanced stage of HCC or other medical contraindications.

In intermediate stage $\mathrm{HCC}$, according to the Barcelona Clinic Liver Cancer staging system (BCLC B) [4], with a multinodular affection transarterial chemoembolization (TACE) is the gold standard therapy to date. TACE was shown in randomized trials to improve survival compared with symptomatic therapy alone, in patients without macrovascular involvement. In advanced stage HCC (BCLC C), including HCC with portal invasion or 
metastasis and refractory disease following TACE, Sorafenib is the only systemic treatment option. [5].

Traditionally, the use of conventional external beam radiation therapy in $\mathrm{HCC}$ treatment was limited due to the low radiation tolerance of the liver especially in patients with underlying disease and a high ChildTurcotte-Pugh score. Yet in the recent years advances in treatment delivery and techniques allowed for enhanced delivery of ablative doses while sparing surrounding critical tissues using stereotactic body radiotherapy (SBRT). Radiation induced liver disease (RILD) after SBRT occurs in fewer than $5 \%$ of cases with careful patient selection [6].

Several prospective and retrospective trials showed promising results in patients treated with SBRT with high rates of local control and acceptable toxicity [7-11]. Thus SBRT could be considered as an alternative to ablation and/or embolization techniques or in cases were these therapies have failed or were contraindicated [12]. Nevertheless there is limited data concerning the safety of SBRT for large tumors or patients with impaired liver function.

In this study we evaluated the efficacy and toxicity of SBRT in large HCC tumors and impaired liver function unsuitable for other treatment options.

\section{Methods}

\section{Patients and treatment characteristics}

This retrospective analysis was approved by the institutional research ethics board. All consecutive patients with HCC, treated with SBRT between 2012 and 2015, who were unsuitable for surgery, RFA or TACE after multidisciplinary board (MDT) decision were enrolled in this analysis and treated according to the same institutional standard operating procedures (SOPs). The MDT panel typically indicated SBRT in patients with very large lesions that progressed or were not suitable for other local treatment modalities taking into account the relative position with respect to organs at risk for SBRT. For patients (BCLC stage $B$ ), with progressive disease (PD) after TACE, SBRT was offered as a local ablative option, as an alternative to systemic therapy with sorafenib, which has a high toxicity profile. Additionally patients with BCLC stage $C$ with PD under sorafenib had no other treatment options.

Diagnosis was established either based on imaging techniques and/or by biopsy [2]. All patients had a good Eastern Cooperative Oncology Group (ECOG) performance status $\leq 2$ with a life expectancy $\geq 6$ months. Clinical examination, blood samples including liver scores and $\alpha$ fetoprotein and the evaluation of the Barcelona-Clinic Liver Cancer (BCLC) classification, Child-Turcotte-Pugh (CTP) score were assessed before SBRT for all patients.

\section{SBRT techniques}

All patients were immobilized in supine position with a customized vacuum cushion (BlueBAG BodyFIX, Innovative Technologies Völp, Innsbruck, Austria) and underwent 4 dimensional-CT (4D CT, Brilliance CT Big Bore, Philips Medical Systems, Cleveland, $\mathrm{OH}$ ). For the 4D acquisition, breathing motion was monitored (Mayo Clinic Respiratory feedback system), including abdominal compression to minimize respiratory motion, using a phase based binning method with ten respiratory phases. The gross tumor volume (GTV) was defined as the arterial phase enhancing lesions with washout in the venous phase and/or delayed phase CT and MRI. Tumor vascular thrombosis (TVT) was also included into the GTV. The internal target volume (ITV) was created accounting for the extent and the position of the tumour at all motion phases in 3 dimensions using the $4 \mathrm{D}-\mathrm{CT}$ image data. The PTV was a uniform $4 \mathrm{~mm}$ expansion of the ITV in all dimensions. Either fiducial markers were implanted or lipiodol depositis, surgical clips, transjugular intrahepatic portosystemic stent shunting (TIPPS) stents were used as fiducial markers for image guided radiotherapy (IGRT). In limited cases where the use of fiducial markers was contraindicated and the tumor was located in the right superior or inferior pole of the liver, the ITV of the borders of the liver was used for IGRT. Patients were treated with 3 to 12 fractions, dependent on the proximity to the organs at risk (OARs) (stomach, small intestine, colon and duodenum) delivered every other day. Three fraction regimens (typically $3 \times 12.5-15$ Gy) were preferred in patients with lesions away from critical structures, 12 fraction regimens (typically $12 \times 4-5.5$ Gy) were preferred in patients with contact to OARs, and 5 fraction regimens (typically $5 \times 7-10 \mathrm{~Gy}$ ) were intermediate in terms of closeness to OARs. For lesions where dose constraints as proposed by Timmerman et al. [13] could not be achieved, we utilized a simultaneous integrated protection (SIP) dose prescription, an intensity modulated radiotherapy (IMRT) technique described in detail elsewhere instead of reducing the dose to the entire PTV [14]. From 2007 to 2013 treatment was prescribed either to the 60 or $80 \%$ encompassing isodose and thereafter according to ICRU report 83 with a $D_{\max }$ of $110-120 \%$. For analysis the prescribed doses were converted to biological effective doses (BED) and equieffective doses for 2 Gy fractions (EQD2), assuming that tumour and late reacting bowel tissue $\alpha / \beta$ ratios were $10 \mathrm{~Gy}$ and 3 Gy, respectively [15].

For all patients a daily on-line correction using cone beam computed tomography (CBCT) scans was applied and oral contrast was given to visualise stomach and/or duodenum in cases of close proximity. 


\section{Toxicity and follow up}

Patients were clinically examined at least weekly during treatment by radiation oncologists. During follow up, complete history, physical examination, blood tests and triphasic liver CTs or MRIs were acquired every three months. Toxicity was scored using the NCI Common Terminology Criteria for Adverse Events v4.03. Radiation induced liver disease (RILD) was defined according to Pan et al. [16] with the typical occurrence between one week to three months after treatment in the absence of PD. All toxicities reported within 3 months after treatment completion were considered as acute; thereafter, any toxicity was considered to be late.

\section{Statistical analysis}

The primary end points were toxicity assessment and local control (LC) in the PTV ('in-field') at 1 year; the latter was defined as the absence of PD within the PTV as per Response Evaluation Criteria in Solid Tumors (RECIST) v1.1 in multiphasic CT or MRI. Lesions that developed or progressed outside the PTV in the liver or lymph nodes were scored as regional PD and those developed in other organs as distant PD. Survival and control times were calculated from the start of SBRT. Time to progression and survival were evaluated with the Kaplan-Meier method. Analyses were performed using SPSS (SPSS Inc., Chicago, IL) Statistical significance was set to $p \leq \underline{0} .05$ and both sided.

\section{Results}

\section{Patients and treatment characteristics}

Between 2013 and 2016, a total of 47 patients with 64 lesions were included in the analysis with a median follow up of for patients alive of 19 months. Patient and Treatment characteristics are summarized in Table 1. Twentythree patients (49\%) had a BCLC stage B and 24 (51\%) patients a stage $C$ at the time of the analysis. Twentyeight patients $(60 \%)$ had a CTP score A $(\mathrm{A} 5=16$, $\mathrm{A} 6=12)$ and $19(40 \%)$ a CTP score $\mathrm{B}(\mathrm{B} 7=10, \mathrm{~B} 8=6$, B9 $=3$ ). Nine $(19 \%)$ patients were diagnosed with oligometastatic disease (lung $=4$, bones $=4$, adrenal $n=2$ ) prior to SBRT, 38 (81\%) patients had multifocal disease confined to the liver and five patients had regional lymph node metastases. Ten patients were treated with SBRT at two different HCC lesions simultaneously and seven patients were pre-treated with SBRT for another HCC-lesion in the past at median time between the two treatments of 5 months. Seventy eight percent received prior and in most cases multiple therapies. Pretreatment included resection (9 patients), radiofrequency ablation (5 patients), sorafenib (10 patients), transarterial chemoembolization (TACE, 34 patients), SBRT (7 patients) and selective internal radiation therapy (SIRT, 2 patients). For the latter a moderate fractionation of $12 \times 4$
Gy was used for the SBRT, mainly due to the lesions size. The first patient had a tumor of $21 \mathrm{~cm}$ in maximum diameter and the second patient two lesions of 11 and $9 \mathrm{~cm}$, respectively. Both patients had a CTP score of A5. No additional constraints were taken into consideration for patients who underwent SIRT or Re-SBRT.

Median GTV was $77 \mathrm{~cm}^{3}$ (interquartile range IQR 37 to $229 \mathrm{ml}$ ) at a median liver volume of $1654 \mathrm{ml}$ (IQR 1384 to $2230 \mathrm{ml}$; Table 1). The median diameter of the lesions was $7 \mathrm{~cm}$ (range 1.7-22, IQR 5-10) $\mathrm{cm}$. Tumor vascular thrombosis (TVT) was present in $13(28 \%)$ patients. Some treatments were planned with inhomogeneous dose distributions: planning target volume encompassing doses were most frequently $80 \%$ (5 lesions, $8 \%$ ) and $60 \%$ (12 lesions, 19\%) of the maximum dose. The median prescribed SBRT dose was 45 (IQR 38-48) Gy in 3 to 12 fractions [see Additional file 1] with a median maximum dose (Dmax) of 53 (IQR 49-59) Gy. One patient discontinued treatment due to esophageal varices bleeding (outside the PTV) after 21 Gy. The median prescribed biological effective dose was $\left(\mathrm{BED}_{10}\right)$ of 76 (IQR 62-86 Gy) and an equivalent dose in 2 Gy fractions (EQD2 10 ) of 63 Gy (IQR 5175 Gy). For IGRT fiducial markers were implanted in 6 patients, lipiodol deposits of prior TACE were used in 34 patients, surgical clips in 1 patient and the TIPPS stents were used in 2 patients. In four cases where the tumor was located in the right superior or inferior pole of the liver, where the implantation of fiducial makers was not possible, the ITV of the borders of the liver was used for IGRT. Twenty-five patients were treated with SIP. Volumetric Arc IMRT was performed in 45 patients.

\section{Local control per lesion and patterns of failure}

Local control at 1 year was $77 \%$ from the start of SBRT (Fig. 1). Overall, seven lesions (11\%) progressed in field between 3 and 17 months after treatment (median 7 months, 95\% CI 5.635-8.365), two in combination with a regional PD (out of field, i.e. liver), two in combination with a distant PD and three of them also in combination with regional and distant metastases. A regional failure in the liver (out of field) was observed in three cases. Sixteen progressed regionally (out of field) in combination with distant metastases and 10 developed only distant metastases (Fig. 2). After PD 11 patients were treated with sorafenib and six patients with TACE and seven patients with regional recurrence were re-irradiated. On univariate analysis the diameter of the tumor and the GTV were associated with improved local control (Table 2). Additionally the EQD2 10 and consecutively $\mathrm{BED}_{10}$ of the prescribed dose, but not the $D_{\max }$ or $D_{95 \%}$, were associated with improved local control (Table 2). The fractionation had no impact on 
Table 1 Patient and treatment characteristics

\begin{tabular}{|c|c|c|c|}
\hline \multirow[t]{2}{*}{ Variable } & All patients & CTP A & CTP B \\
\hline & No. (\%) & No. (\%) & No. (\%) \\
\hline No. of patients & $47(100 \%)$ & $28(60 \%)$ & $19(40 \%)$ \\
\hline \multicolumn{4}{|l|}{ Age(years) } \\
\hline Median (range) & $69(29-84)$ & $70(45-84)$ & $69(29-83)$ \\
\hline \multicolumn{4}{|l|}{ BCLC stage } \\
\hline B & $23(49 \%)$ & $14(50 \%)$ & $9(47 \%)$ \\
\hline$C$ & $24(51 \%)$ & $14(50 \%)$ & $10(53 \%)$ \\
\hline \multicolumn{4}{|l|}{ CTP score } \\
\hline A & $28(60 \%)$ & & \\
\hline A5 & $16(34 \%)$ & 16 & \\
\hline A6 & $12(26 \%)$ & 12 & \\
\hline B & $19(40 \%)$ & & \\
\hline B7 & $10(21 \%)$ & & 10 \\
\hline B8 & $6(13 \%)$ & & 6 \\
\hline B9 & $3(6 \%)$ & & 3 \\
\hline $\begin{array}{l}\text { Underlying liver } \\
\text { disease }\end{array}$ & $41(87 \%)$ & & \\
\hline Hepatitis B & $4(9 \%)$ & $3(11 \%)$ & $1(5 \%)$ \\
\hline Hepatitis C & $6(13 \%)$ & $3(11 \%)$ & $3(16 \%)$ \\
\hline Alcohol related & $24(51 \%)$ & $11(39 \%)$ & $12(63 \%)$ \\
\hline $\mathrm{NASH}^{\mathrm{a}}$ & $6(13 \%)$ & $5(18 \%)$ & $2(11 \%)$ \\
\hline $\begin{array}{l}\text { Primary biliary } \\
\text { cirrhosis }\end{array}$ & $1(2 \%)$ & $1(7 \%)$ & $0(0 \%)$ \\
\hline None & $6(13 \%)$ & $5(18 \%)$ & $1(5 \%)$ \\
\hline \multicolumn{4}{|l|}{ Previous treatments ${ }^{g}$} \\
\hline Surgery & $9(19 \%)$ & $8(29 \%)$ & $4(21 \%)$ \\
\hline TACE $^{\mathrm{b}}$ & $34(72 \%)$ & $21(75 \%)$ & $13(68 \%)$ \\
\hline RFA $^{c}$ & $5(11 \%)$ & $5(18 \%)$ & $1(5 \%)$ \\
\hline Sorafenib & $10(21 \%)$ & $8(29 \%)$ & $2(11 \%)$ \\
\hline $\mathrm{SIRT}^{\mathrm{d}}$ & $2(4 \%)$ & $2(7 \%)$ & $0(0 \%)$ \\
\hline $\mathrm{SBRT}^{\mathrm{e}}$ & $7(15 \%)$ & $2(7 \%)$ & $3(16 \%)$ \\
\hline $\begin{array}{l}\text { Tumor vascular } \\
\text { thrombosis }\end{array}$ & $13(28 \%)$ & $5(18 \%)$ & $8(42 \%)$ \\
\hline Extrahepatic disease & $9(19 \%)$ & $6(21 \%)$ & $3(16 \%)$ \\
\hline $\begin{array}{l}\text { Multiple lesions at } \\
\text { baseline }\end{array}$ & $39(83 \%)$ & $24(86 \%)$ & $15(79 \%)$ \\
\hline \multicolumn{4}{|l|}{ GTV volume $e^{f}$} \\
\hline Median (IQR) $\mathrm{cm}^{3}$ & $77(37-229)$ & $69(35-214)$ & $108(38-268)$ \\
\hline \multicolumn{4}{|c|}{ Diameter of the lesions ${ }^{f}$} \\
\hline Media(IQR) cm & $7(5-10)$ & $7(4-10)$ & $8(5-10)$ \\
\hline \multicolumn{4}{|l|}{ Liver Volume } \\
\hline Median (range) $\mathrm{cm}^{3}$ & $\begin{array}{l}1654 \\
(1384-2230)\end{array}$ & $\begin{array}{l}1492 \\
(1340-2277)\end{array}$ & $\begin{array}{l}1819 \\
(1554-1997)\end{array}$ \\
\hline \multicolumn{4}{|l|}{ Prescription dose $(G y)^{f}$} \\
\hline Median (IQR) & $45(38-48)$ & $45(38-48)$ & $45(44-50)$ \\
\hline
\end{tabular}

Table 1 Patient and treatment characteristics (Continued)

\begin{tabular}{|c|c|c|c|}
\hline \multicolumn{4}{|l|}{ Dmax } \\
\hline Median (IQR) & $53(49-59)$ & $54(52-59)$ & $51(48-58)$ \\
\hline \multicolumn{4}{|l|}{ D95\% } \\
\hline Median (IQR) & $45(38-48)$ & $45(37-48)$ & $45(44-50)$ \\
\hline \multicolumn{4}{|c|}{ EQD2 10 prescribed $(G y)^{f}$} \\
\hline Median (IQR) & $63(51-75)$ & $56(50-71)$ & $71(56-85)$ \\
\hline \multicolumn{4}{|l|}{ EQD2 10 Dmax } \\
\hline Median (IQR) & $86(62-104)$ & $91(62-104)$ & 82 (61-99) \\
\hline \multicolumn{4}{|l|}{ EQD2 10 D95\% } \\
\hline Median (IQR) & $60(52-76)$ & $56(48-72)$ & $71(56-85)$ \\
\hline \multicolumn{4}{|c|}{ BED $_{10}$ prescribed $(G y)^{f}$} \\
\hline Median (IQR) & $76(62-86)$ & $67(61-85)$ & $86(67-102)$ \\
\hline \multicolumn{4}{|l|}{ BED $_{10}$ Dmax } \\
\hline Median (IQR) & $102(75-125)$ & $109(75-125)$ & $99(73-115)$ \\
\hline \multicolumn{4}{|l|}{$\mathrm{BED}_{10} \mathrm{D} 95 \%$} \\
\hline Median (IQR) & 72 (62-91) & $67(57-86)$ & $86(67-102)$ \\
\hline \multicolumn{4}{|c|}{ Mean liver dose (Gy) } \\
\hline Median (IQR) & $17.9(8.7-25)$ & $19(9.8-25)$ & $16.5(7.8-22)$ \\
\hline \multicolumn{4}{|c|}{ EQD22, mean liver dose } \\
\hline Median (IQR) & $16.5(9.5-23)$ & $17.2(9-23.5)$ & $15.6(8.5-20.5)$ \\
\hline
\end{tabular}

${ }^{a}$ nonalcoholic fatty liver disease

'TACE: transarterial chemoembolization

'RFA: radiofrequency ablation

${ }^{d}$ SIRT: selective internal radiation therapy

eSBRT: stereotactic body radiotherapy

fper lesion

${ }^{g}$ some patients had more than one treatments previous to SBRT $I Q R$ inter-quartile range

local control and there was no difference concerning LC between patients treated with or without simultaneous integrated protection (SIP, $p=0.944$, log rank, Table 2). The median progression free survival time was 7 months (95\% CI, 5.3-8.6).

\section{Overall survival}

Median overall survival time was 9 months (95\% CI, 7.715-10.285). The only significant factor for OS on univariate analysis was the volume of the GTV (Table 2). At the time of the analysis 31 patients $(66 \%)$ had died, 12 due to disease progression, three of whom due to liver decompensation (3, 6, 10 months after treatment). One patient died due to liver decompensation without PD 4 months after SBRT. Thirteen patients died due other causes (upper gastrointestinal bleed at distance from the PTV $n=3$, pneumonia $n=2$, stroke $n=1$, pulmonary decompensation $n=1$, urosepsis $n=1$, cardiac 


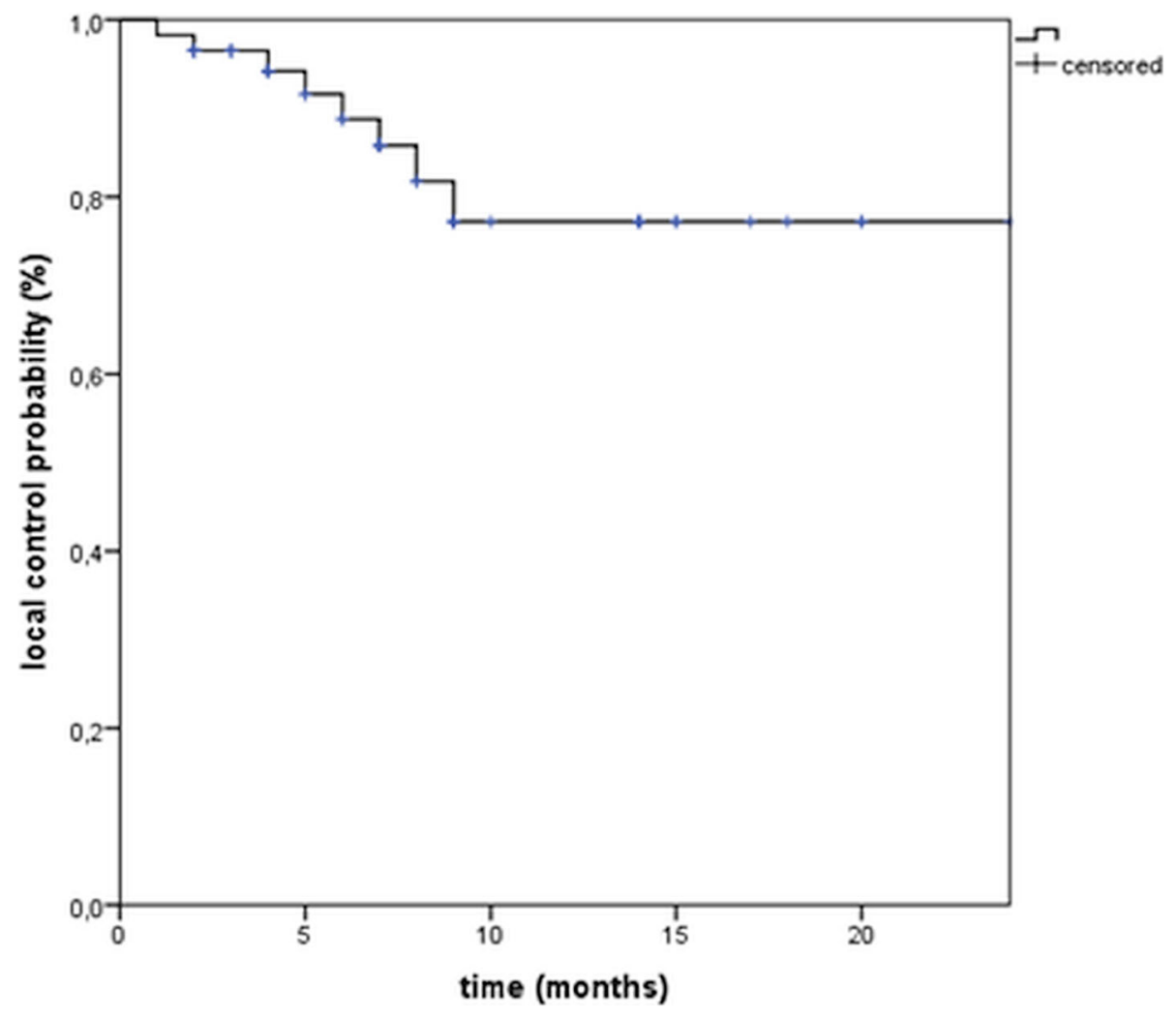

Fig. 1 Local control from the time of radiotherapy

decompensation $n=1$, sepsis due to liver abscess $n=1$, renal failure $n=3$ ) and five due to unknown causes. The presence of extrahepatic disease had no impact on overall survival ( $n=9$ patients, median OS 9 vs 10 months, $p=0.4 \log$ rank, Table 2).

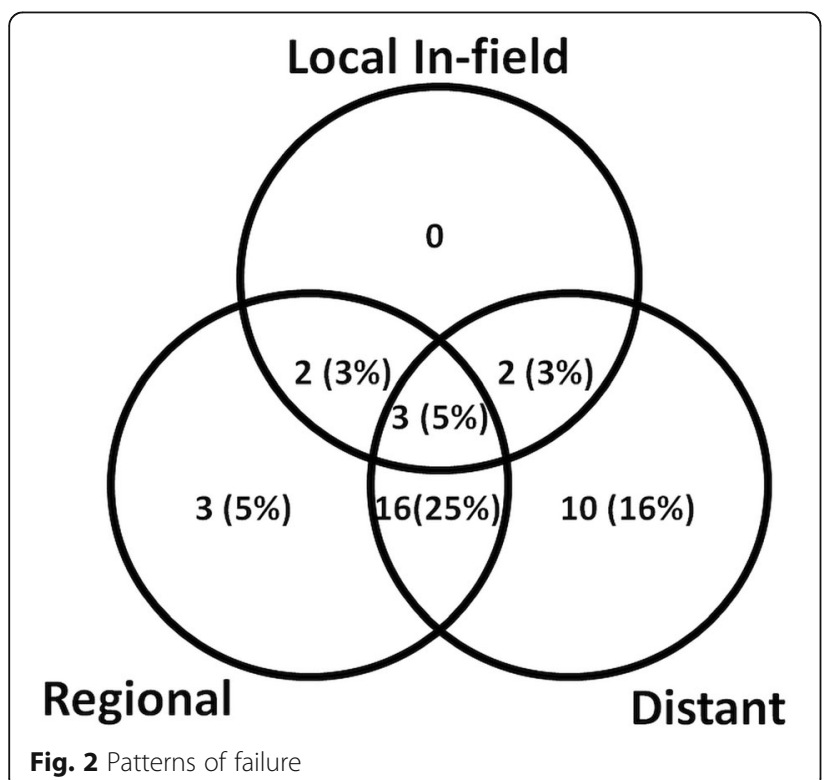

\section{Toxicity}

Three patients with known portal hypertension prior to therapy developed gastric ulcers with bleeding CTC grade $2-3$, one, three, four and five months after SBRT which were treated with proton pump inhibitors (2 patients, grade 2 ) and transfusion (1 patient, grade 3 ). In the first case the patient who was treated in the past with liver SBRT for another HCC lesion, with an interval of 4 months between the two treatments, developed CTC grade 3 gastroduodenitis requiring a transfusion four months after the second treatment. The Dmax and D $0.5 \mathrm{~cm}^{3}$ at the stomach at first treatment were $46.6 \mathrm{~Gy}$ (EQD2 $\left.2_{3} 64 \mathrm{~Gy}\right)$ and $44.8 \mathrm{~Gy}\left(\mathrm{EQD} 2_{3} 60 \mathrm{~Gy}\right)$ in 12 fractions and in the second treatment 14Gy (EQD2 3 $12.7 \mathrm{~Gy})$ and $13.4\left(\mathrm{EQD} 2_{3} 12 \mathrm{~Gy}\right)$ in 9 fractions. In this case the addition of the second SBRT contributed probably to the development of the gastrointestinal bleeding. In case of the second patient the Dmax was $33.8 \mathrm{~Gy}$, $\mathrm{D} 0.5 \mathrm{~cm}^{3} 31.7 \mathrm{~Gy}, \mathrm{D} 5 \mathrm{~cm}^{3} 28.1 \mathrm{~Gy}$ in five fractions (corresponding EQD2 3 values 66 Gy, 59.2 Gy, 48.4 Gy). All constraints were respected except of the Dmax, which exceeded the constraints by $0.1 \mathrm{~Gy}$. The third patient had a Dmax of 28.8 Gy in 5 fractions, D0.5 cc 25.3 and D5cc 20 Gy (corresponding EQD2 3 50.4 Gy, 41 Gy and $28 \mathrm{~Gy}$ ). The latter had a known gastric antral vascular ectasia (GAVE) which is an uncommon cause of 
Table 2 Univariate analysis for local control and overall survival

\begin{tabular}{|c|c|c|c|c|c|c|}
\hline \multirow[t]{2}{*}{ Variable } & \multicolumn{3}{|c|}{ Local control } & \multicolumn{3}{|c|}{ Overall survival } \\
\hline & $\mathrm{HR}$ & $95 \% \mathrm{Cl}$ & $p$ & $\mathrm{HR}$ & $95 \% \mathrm{Cl}$ & $p$ \\
\hline CTP & 1.725 & $(0.406-7.335)$ & 0.5 & 1.806 & $(0.884-3.691)$ & 0.1 \\
\hline Multifocality & 0.696 & $(0.085-5.688)$ & 0.7 & 1.471 & $(0.512-4.228)$ & 0.5 \\
\hline Diameter & 1.147 & $(1.008-1.305)$ & 0.04 & 1.017 & $(0.952-1.086)$ & 0.6 \\
\hline Extrahepatic disease & n.a. & & & 0.653 & $(0.260-1.640)$ & 0.7 \\
\hline Fractionation & 0.919 & $(0.755-1.118)$ & 0.4 & 0.952 & $(0.860-1.053)$ & 0.3 \\
\hline SIP & 0.950 & $(0.227-3.989)$ & 0.9 & 1.298 & $(0.639-2.637)$ & 0.5 \\
\hline GTV Volume & 1.001 & $(1.000-1.002)$ & 0.008 & 1.001 & $(1.000-1.001)$ & 0.008 \\
\hline TVT & 0.977 & (0.189-5.058) & 0.9 & 1.674 & $(0.781-3.590)$ & 0.2 \\
\hline EQD2 10 , prescribed & 0.952 & (0.907-0.998) & 0.04 & 1.002 & $(0.978-1.026)$ & 0.9 \\
\hline EQD2 10, Dmax & 0.992 & $(0.967-1.017)$ & 0.5 & 0.998 & (0.988-1.008) & 0.7 \\
\hline EQD210, D95\% & 0.978 & (0.919-1.014) & 0.4 & 1.013 & $(0.990-1.036)$ & 0.3 \\
\hline $\mathrm{EQD2} 2$, mean liver dose & n.a. & & & 1.009 & $(0.967-1.054)$ & 0.6 \\
\hline $\mathrm{BCLC}$ & n.a. & & & 0.868 & $(0.428-1.761)$ & 0.7 \\
\hline
\end{tabular}

CTP Child-Turcotte-Pugh score, TVT Tumor vascular thrombosis, UVA univariate analysis, OS Overall survival, LC Local control, CI Confidence interval, n.a. not applicable

Dose, Diameter and GTV were considered as continuous variables

Table 3 Toxicities, CTCAE $>2$

\begin{tabular}{|c|c|c|c|c|}
\hline \multirow[t]{2}{*}{ A.Toxicitiy } & Grade 2 & Grade 3 & Grade 4 & Grade 5 \\
\hline & No (\%) & No (\%) & No (\%) & No (\%) \\
\hline \multicolumn{5}{|l|}{ Biochemical } \\
\hline ALT/AST (u/l) & 0 & 0 & 0 & \\
\hline Bilirubin (mg/dl) & $2(4 \%)$ & $6(13 \%)$ & 0 & \\
\hline INR & 0 & 0 & 0 & \\
\hline $\mathrm{AP}(\mathrm{U} / \mathrm{I})$ & $1(2 \%)$ & 0 & 0 & \\
\hline GGT (U/l) & $2(4 \%)$ & 0 & 0 & \\
\hline \multicolumn{5}{|l|}{ Gl-Toxicitiy } \\
\hline Gastrioduodenitis / Gl bleeding & $2(4 \%)$ & $1(2 \%)$ & 0 & \\
\hline \multicolumn{5}{|l|}{ Liver-Toxicity } \\
\hline Abscess & & & $1(2 \%)$ & \\
\hline RILD & & $1(2 \%)^{b}$ & & \\
\hline Dekompensation & & & & $1(2 \%)$ \\
\hline B. & \multicolumn{4}{|c|}{ CTP Deterioration ${ }^{\mathrm{a}}$ No (\%) } \\
\hline \multicolumn{5}{|l|}{ Score } \\
\hline 1 Point & 4 & & & \\
\hline 2 Points & $1(2 \%)^{b}$ & & & \\
\hline Class & $2(4 \%)$ & & & \\
\hline
\end{tabular}

Gl gastrointestinal, CTCAE Commom Terminology Criteria for Adverse Events (CTCAE) version 4.0, INR international normalized ratio, AST Alanine aminotransferase, ALT Aspartate aminotransferase, AP Alkaline phosphatase, GGT Gamma-Glutamyltransferase, RILD radiation induced liver desease ${ }^{a}$ without progressive disease

bame patient chronic gastrointestinal bleeding. In this case the constraints did not exceed the constraints proposed by Timmerman et al. [13]. An increase of the CTP score was observed in 17 patients of whom 12 patients had $\mathrm{PD}$. Of the five patients without progression $4 \mathrm{had}$ an increase of one point (B7 to B8, A6 to B7, A5 to A6, B8 to B9) and 1 developed an increase of $\geq 2$ points after treatment (A6 to B8) due to a radiation induced liver disease (RILD). The latter recovered fully and died 9 months after SBRT due to renal failure. Only one of these patients, with an increase of one point (A5 to A6) died due to liver decompensation without disease progression 4 months after SBRT. This patient had a $6.6 \mathrm{~cm}$ tumor and was treated in 5 fractions with a median dose to the liver of 16 Gy. There were no significant alkaline phosphatase elevations or liver transaminases observed. None of the dose constraints for the liver were violated. One patient developed a necrotic abscess in the PTV of the liver due to a dislocation of a pre-existing stent of the bile duct (Table 3).

\section{Discussion}

SBRT can lead to excellent results for small HCC tumors (Table 4), but there is little experience with larger tumors. Delivering high doses to large HCCs can be very challenging due to the higher mean liver doses irradiated, that often compromise the liver constraints and thus increase the risk for liver failure, especially in a collective which is highly pre-treated or with a higher Child-Pugh score. As reported by Crane et al. [19] the vast majority of physicians interpret SBRT as meaning 
Table 4 Review of Literature

\begin{tabular}{|c|c|c|c|c|c|c|c|c|}
\hline Author & Study & $\begin{array}{l}\text { Nr. of } \\
\text { patients }\end{array}$ & СTP & Diameter $(\mathrm{cm})^{a}$ & Fractionation & LC@1 year & mOS & Toxicity \\
\hline Mendez Romero [23] & Pr. & $\begin{array}{l}5 \\
2\end{array}$ & $\begin{array}{l}A \\
B\end{array}$ & 4.7 & $3-5 \times 5-12$ Gy & $75 \%$ & 22 & 1 lethal liver failure \\
\hline Tse [34] & Pr. & 31 & A & $173 \mathrm{~cm}^{3 \mathrm{~b}}$ & $6 \times 4-9$ Gy & $65 \%$ & 11.7 & $\begin{array}{l}8 \text { grade } 3 \text { enzyme elevations, } \\
1 \text { pulmonary embolism, } 1 \\
\text { tumor-duodenal connection }\end{array}$ \\
\hline Jang [35] & Ret & $\begin{array}{l}74 \\
8\end{array}$ & $\begin{array}{l}\text { A } \\
B\end{array}$ & 3 & $3 \times 11-20 G y$ & $\begin{array}{l}87 \% @ \\
2 y .\end{array}$ & $\begin{array}{l}63 \% @ \\
2 y .\end{array}$ & $\begin{array}{l}5 \text { Gl toxicity grade } 3 \\
6 \text { CTP elevation }>2\end{array}$ \\
\hline Huang [36] & Ret & $\begin{array}{l}23 \\
4 \\
1\end{array}$ & $\begin{array}{l}A \\
B \\
C\end{array}$ & 4.4 & $\begin{array}{l}10 \times 4.5 \text { Gy } \\
18-20 \times 2.5 \text { Gy } \\
18-20 \times 1.8 \text { Gy }\end{array}$ & $87.6 \%$ & 23 & 1 grade 3 gastric ulcer \\
\hline Bae [37] & Ret & $\begin{array}{l}18 \\
2\end{array}$ & $\begin{array}{l}\text { A } \\
B\end{array}$ & $\begin{array}{l}<3 \mathrm{~cm}(80 \%) \\
3-5 \mathrm{~cm}(20 \%)\end{array}$ & $5 \times 10 \mathrm{~Gy}$ & $85 \%$ & $\begin{array}{l}100 @ \\
1 y .\end{array}$ & No grade 3 toxicities \\
\hline Jung [38] & Ret & $\begin{array}{l}68 \\
24\end{array}$ & $\begin{array}{l}A \\
B\end{array}$ & $8.6 \mathrm{~cm}^{3 \mathrm{~b}}$ & $3-4 \times 10-20 G y$ & $\begin{array}{l}92 \% @ \\
3 y .\end{array}$ & & 6 patients grade 3 RILD \\
\hline Wahl [12] & Ret. & $\begin{array}{l}57 \\
24 \\
2\end{array}$ & $\begin{array}{l}A \\
B \\
C\end{array}$ & $\begin{array}{l}<2 \mathrm{~cm}(48 \%) \\
2-3 \mathrm{~cm}(26 \%) \\
3-5 \mathrm{~cm}(23 \%) \\
>5 \mathrm{~cm}(3.7 \%)\end{array}$ & $3-5 \times 6-10 G y$ & $97.4 \%$ & $\begin{array}{l}74 \% @ \\
1 y .\end{array}$ & $\begin{array}{l}1 \text { RILD, } 1 \text { Gl bleeding } \\
1 \text { worsening ascites }\end{array}$ \\
\hline Andolino [9] & Ret. & $\begin{array}{l}36 \\
24\end{array}$ & $\begin{array}{l}A \\
B\end{array}$ & $3.1 \mathrm{~cm}$ & 3-5 × 8-16Gy & $\begin{array}{l}90 \% @ \\
2 y .\end{array}$ & $\begin{array}{l}48 \% @ \\
2 y .\end{array}$ & $20 \%$ CTP progression \\
\hline Bibault [22] & Ret. & $\begin{array}{l}66 \\
9\end{array}$ & $\begin{array}{l}A \\
B\end{array}$ & $3.7 \mathrm{~cm}$ & $3 \times 8-15 G y$ & $89.8 \%$ & 15 & $\begin{array}{l}5 \text { liver decompensations, } \\
1 \text { grade } 4 \text { gastric ulcer, } \\
3 \text { grade } 2 \text { duodenal } \\
\text { ulcers }\end{array}$ \\
\hline Huertas [21] & Ret. & $\begin{array}{l}76 \\
11\end{array}$ & $\begin{array}{l}\text { A } \\
B\end{array}$ & $2.4 \mathrm{~cm}$ & $3 \times 15 G y$ & $99 \%$ & $\begin{array}{l}82 \% @ \\
1 y .\end{array}$ & $\begin{array}{l}1 \text { grade } 5 \text { hematemesis } \\
2 \text { grade }>3 \text { gastric ulcers }\end{array}$ \\
\hline Scorsetti [24] & Ret. & $\begin{array}{l}23 \\
20\end{array}$ & $\begin{array}{l}A \\
B\end{array}$ & $4.8 \mathrm{~cm}$ & $\begin{array}{l}3 \times 16-25 \mathrm{~Gy} \\
6 \times 6-10 \mathrm{~Gy}\end{array}$ & $86 \%$ & 18 & $\begin{array}{l}7 \text { grade }>3 \text { liver enzyme } \\
\text { elevations }\end{array}$ \\
\hline Seo [39] & Ret. & $\begin{array}{l}34 \\
4\end{array}$ & $\begin{array}{l}A \\
B\end{array}$ & $40.5 \mathrm{ml}^{\mathrm{b}}$ & $3 \times 11-12 \mathrm{~Gy}$ & $79 \%$ & 32 & 1 grade 3 soft tissue toxicity \\
\hline Kwon [40] & Ret. & $\begin{array}{l}38 \\
4\end{array}$ & A & $15.4 \mathrm{ml} \mathrm{l}^{\mathrm{b}}$ & $3 \times 10-13 G y$ & $72 \%$ & $\begin{array}{l}93 \% @ \\
1 \text { y. }\end{array}$ & $\begin{array}{l}1 \text { radiation induced hepatic } \\
\text { failure }\end{array}$ \\
\hline Takeda [41] & Ret. & $\begin{array}{l}14 \\
2\end{array}$ & $\begin{array}{l}\text { A } \\
B\end{array}$ & $1.9-7 \mathrm{~cm}$ & $5-7 \times 5-10 G y$ & $100 \%$ & $100 \%$ & 1 RILD \\
\hline Price [42] & Ret. & $\begin{array}{l}14 \\
12\end{array}$ & $\begin{array}{l}A \\
B\end{array}$ & $\max .6 \mathrm{~cm}$ & $3-5 \times 8-16 G y$ & $97 \%$ & $77 \%$ & 20\% CTP worsening \\
\hline Kang [43] & Pr. & $\begin{array}{l}41 \\
6\end{array}$ & $\begin{array}{l}A \\
B\end{array}$ & $2.9 \mathrm{~cm}$ & $3 \times 14-20 G y$ & $\begin{array}{l}94 \% @ \\
2 y .\end{array}$ & $\begin{array}{l}68.7 @ \\
2 y .\end{array}$ & $\begin{array}{l}3 \text { grade } 3 \mathrm{Gl} \text { toxicity, } \\
2 \text { grade } 4 \text { gastric ulcers }\end{array}$ \\
\hline Su [44] & Ret & $\begin{array}{l}114 \\
18\end{array}$ & $\begin{array}{l}A \\
B\end{array}$ & $1.1-5.0 \mathrm{~cm}$ & $\begin{array}{l}1 \times 28-30 \text { Gy } \\
42-46 \text { Gy in } 3-5 \\
\text { fractctions }\end{array}$ & $90 \%$ & & $\begin{array}{l}11 \text { patients hepatic toxicity } \\
\text { grade } \geq 3\end{array}$ \\
\hline Kang [45] & Ret & $\begin{array}{l}67 \\
34\end{array}$ & $\begin{array}{l}A \\
B\end{array}$ & n.s. & 6 fractions & 20-29.4@ 2y & $12-15$ & $\begin{array}{l}25 \text { cases deteriorated from } \\
\text { grade } \\
A \text { to } B, 4 \text { from } A \text { to } C \text { and } 6 \\
\text { from } B \text { to } C\end{array}$ \\
\hline Sanuki [46] & Ret & $\begin{array}{l}158 \\
27\end{array}$ & $\begin{array}{l}A \\
B\end{array}$ & $2.7(0.8-5) \mathrm{cm}$ & $5 \times 7-8 \mathrm{~Gy}$ & 91\%@3y. & 70\%@3y. & $\begin{array}{l}13 \% \text { acute grade }>3,2 \text { grade } \\
5 \text { liver failure }\end{array}$ \\
\hline Kimura [47] & Ret & $\begin{array}{l}56 \\
9\end{array}$ & $\begin{array}{l}A \\
B\end{array}$ & $1.6 \mathrm{~cm}$ & $4 \times 12$ Gy & 100\%@2y. & 76\%@ 2 y. & $23 \%$ grade $>3$ \\
\hline Weiner [26] & Pr. & 12 & $A, B$ & ca. $5 \mathrm{~cm}$ & $5 \times 8-11$ Gy & $91 \%$ & $\begin{array}{l}38 \% @ \\
1 \text { y. }\end{array}$ & $\begin{array}{l}9 \text { CTP decline } \\
2 \text { grade 5hepatic failure }\end{array}$ \\
\hline Que [48] & Ret & $\begin{array}{l}104 \\
11\end{array}$ & $\begin{array}{l}A \\
B\end{array}$ & $\begin{array}{l}\leq 4 \mathrm{~cm} \mathrm{(35 \% )} \\
4-9 \mathrm{~cm} \mathrm{(41 \% )} \\
\geq 10 \mathrm{~cm} \mathrm{(24 \% )}\end{array}$ & $\begin{array}{l}26-40 \text { Gy in } 3-5 \\
\text { fract. }\end{array}$ & $85 \%$ & 15 & $\begin{array}{l}3 \text { patients grade } 5 \\
25 \text { patients grade } 3\end{array}$ \\
\hline Que [27] & Ret. & 22 & A & $11.4 \mathrm{~cm}$ & $5 \times 5.2-8 G y$ & $55.6 \%$ & 11 & 1 grade 3 liver enzyme \\
\hline
\end{tabular}


Table 4 Review of Literature (Continued)

\begin{tabular}{|c|c|c|c|c|c|c|c|c|}
\hline & & 2 & B & & & & & elevation \\
\hline Bujold [10] & Pr. & $\begin{array}{l}102 \\
0\end{array}$ & $\begin{array}{l}A \\
B\end{array}$ & $7.2 \mathrm{~cm}$ & 6 × 4-9Gy & $88 \%$ & 17 & $\begin{array}{l}6 \text { grade }>3 \text { liver } \\
\text { failures, } 1 \text { grade } 5 \\
\text { cholangitis, } 1 \text { grade } \\
5 \text { Gl bleed } \\
16 \text { grade } \geq 3 \text { enzyme } \\
\text { elevations }\end{array}$ \\
\hline Culleton [33] & $\begin{array}{l}\text { Pr./ } \\
\text { Ret. }\end{array}$ & $\begin{array}{l}0 \\
29\end{array}$ & $\begin{array}{l}A \\
B\end{array}$ & $5.1 \mathrm{~cm}$ & $\begin{array}{l}5-15 \text { fractions } \\
19.7-46.8 \mathrm{~Gy}^{\mathrm{c}}\end{array}$ & n.a. & 7.9 & $\begin{array}{l}63 \% \text { CTP decline } \geq 2 \\
\text { points, } 5 \text { grade } 3 \\
\text { thrombocytopenia, } \\
3>\text { grade } 3 \text { elevation } \\
\text { of liver enzymes }\end{array}$ \\
\hline Current study & Ret & $\begin{array}{l}28 \\
19\end{array}$ & $\begin{array}{l}A \\
B\end{array}$ & $7 \mathrm{~cm}$ & $3-12 \times 4-15 G y$ & $77 \%$ & 9 & $\begin{array}{l}1 \text { RILD, } 1 \text { grade } 5 \text { liver } \\
\text { decompensation, } 1 \text { grade } \\
3 \text { Gl-bleed, } \\
1 \text { necrotic abscess }\end{array}$ \\
\hline
\end{tabular}

doses of radiation (range, 4-20 Gray [Gy]) that may not be ablative but are delivered within about 1 week (i.e., in 3-6 fractions). Adherence to this approach has limited the effectiveness of SBRT for large liver tumors $(>7 \mathrm{~cm})$ because of the need to reduce doses to meet organ constraints. Similar to the ongoing LungTech trial delivering eight fractions for central lung tumors (EORTC-2211308113, EudraCT Number 2012-000415-83) which might be considered as hypofractionation rather than SBRT we therefore chose to use up to 12 fractions. However this allowed the delivering a higher $\mathrm{BED}_{10}$ compared to others studies. In a study by Que. et al. [20] reporting results on large HCC (median diameter $11.4 \mathrm{~cm}$ ) patients were treated with 5 fractions of 5.2-8 Gy resulting to an EQD $2_{10}$ of 33-60 Gy. The local control was modest with $55.5 \%$ at 1 year as well as toxicities (1 grade 3 liver enzyme elevation) but only 2 of the 22 patients had a CTP score B. In a prospective Phase I/II study by Bujold et al. tumors with a median size of $7.2 \mathrm{~cm}$ were treated with a total dose of 24-54 in 6 fractions with an EQD2 10 ranging between 28 and 85.5 Gy (median 48 Gy). They reported local control rates of $88 \%$ at 1 year. Although all patients had a CTP score A, a CTP score deterioration occurred in $46 \%$ of the patients and there were 5 (5\%) grade 5 liver failures. In another prospective study from the Princes Margaret Cancer Center, Culleton et al. assessed the outcome of patients treated with SBRT with Child-Pugh B or C HCC (median diameter $5.1 \mathrm{~cm}$ ), unsuitable for liver transplantation. They treated 29 patients with CTP B HCCs with a median dose of 30 Gy in 6 fractions (median EQD2 $2_{10}: 37.5$ Gy) and reported high toxicity rates with a decline in CP score by $>2$ points at 3 months in $63 \%$ of the patient. They concluded that SBRT is a treatment option for selected
HCC patients with small HCCs and modestly impaired (CP B7) liver function.

Compared with the study of Bujold et al. we could show favorable local control rates of $77 \%$ at one year (1-y-LCR), Table 4, [9], [12], [21-25] with a lower toxicity profile although the tumors were of similar size, the EQD $2_{10}$ delivered was higher, the Child-Pugh score was higher and the patients were heavily pre-treated including re-SBRT and SIRT. This is probably due to the use of a more moderate fractionation, without compromising the dose constraints for the OARs and in cases were the constraint could not be met, the use of SIP, that allowed the delivery of a lower dose to small subvolumes without compromising the dose to the hole PTV, while staying within tolerance of the OARs. Neither the use of SIP nor the use of 12 fractions had a negative impact on local control or overall survival. In this way we achieved a higher median EQD2 10 : 63 Gy when compared with other groups treating large HCCs with more moderate toxicities. Similar to our approach, Crane et al. [19], suggested a simultaneous integrated boost (SIB) with simultaneous integrated protection (SIP) for large liver tumors treated with SBRT.

The overall survival in our analysis was intermediate due to several factors such as multiple pre-treatments (resection, TACE, sorafenib, SBRT, SIRT), the presence of a CTP score B in $40 \%$ at the time of treatment, the presence of tumor vascular thrombosis in $28 \%$ and the presence of extrahepatic disease in $19 \%$.

In a propensity score matching comparing long term survival between patients treated either with transarterial or surgical resection for huge hepatocellular carcinoma [28] the one year survival rate in the surgery group was $69.7 \%$ and in the TACE group 
$40.2 \%$ which is similar to our data. For patients with oligometastatic disease (BCLC stage C) there is emerging evidence that TACE, a local ablative treatment, significantly improves OS $[17,18]$, Bettinger submitted). Taking these data into consideration, patients that progressed under TACE were also offered SBRT by the MDT panel, as an alternative to systemic treatment. Furthermore, according to Crane et al. [19], the rationale for taking an aggressive approach to treating large liver tumors is that patients often die from liver failure related to disease progression regardless of the presence of extrahepatic disease. As all recurrences observed in our analysis occurred in combination with either regional progression in the liver or distant progression, combining Sorafenib with SBRT, aiming to enhance the efficacy of SBRT while treating extrahepatic disease, seemed promising. This hypothesis was investigated in a phase I trial by the group of the Princess Margaret Cancer Center, $[29,30]$. The concurrent use of sorafenib with SBRT resulted in unacceptably high rates of serious toxicity and is not recommended for further testing, particularly in patients in whom irradiation of a large amount of liver. Currently a randomized phase III trial (RTOG 1112) is testing the role of SBRT followed by sorafenib vs sorafenib alone in terms of overall survival improvement.

A number of limitations applying to our study need to be considered, including the retrospective and singleinstitution nature of our study and the small sample size tested. As such, these results should be interpreted with caution and only hypothesis generating.

In the pre SBRT era radiotherapy was considered a treatment with high toxicity rates due to the underlying liver disease in most of the patients. Today, there is growing evidence $[10,31]$ that SBRT for hepatocellular carcinoma is a safe treatment option with toxicity rates ranging from 0 to $36 \%[9,10]$ and radiation induced liver disease is less common $<5 \%$ in experienced hands.

Furthermore radiotherapy is a very well tolerated treatment in terms of quality of life [31,32] with the only observed deficits being temporary worsening of appetite and fatigue. In the prospective study of Klein et al. [31] overall quality of life did not decline and baseline overall QOL predicted improved survival. Stereotactic body radiation therapy is well tolerated and warrants comparison against other liver-directed therapies.

\section{Conclusion}

In conclusion, our results are highly concordant with published literature regarding local control for smaller tumors. We could show a good overall toxicity profile with only a slightly shorter overall survival in patients with high CTP score which is an unfavorable prognostic factor [33], pre-treatment, multifocality, frequent TVT and tumor volume. Furthermore we have also included patients which were re-irradiated and tolerated SBRT without major complications. SBRT is a feasible treatment for and warrants greater recognition as a treatment option in the management of this malignancy. In this context we are now conducting, a phase II single institutional prospective comparison between TACE and SBRT (HERACLES, DRKS number: DRKS00008566).

\section{Additional file}

Additional file 1: Supplementary table showing the fractionation

regimes used. (DOCX $13 \mathrm{~kb}$ )

\begin{abstract}
Abbreviations
4D CT: 4 dimensional-CT; BCLC: Barcelona-Clinic Liver Cancer; BED: Biological effective doses; CBCT: Cone beam computed tomography; CTP: Child-TurcottePugh; ECOG: Eastern cooperative oncology group; EQD2: Equieffective doses for 2 Gy fractions; GTV: Gross tumor volume; HCC: Hepatocellular carcinoma; IMRT: Intensity modulated radiotherapy; ITV: Internal target volume; LC: Local control; MDT: Multidisciplinary board; OARs: Organs at risk; RECIST: Response evaluation criteria in solid tumors; RFA: Radiofrequency ablation; RILD: Radiation induced liver disease; SBRT: Stereotactic body radiotherapy; SIP: Simultaneous integrated protection; SIRT: Selective internal radiation therapy; SOPs: Standard operating procedures; TACE: Transarterial chemoembolization
\end{abstract}

\section{Acknowledgements}

Not applicable.

Funding

None.

Availability of data and materials

The datasets supporting the conclusions of this article are included within the article.

\section{Authors' contributions}

EG, TB participated in the study design, contributed to the data collection, and draft the manuscript. LH contributed to the data collection. UN, ALG made important contributions in revising the content. All authors made contributions in revising the content, read and approved the final manuscript.

Ethics approval and consent to participate

The study was evaluated by the ethics committee of the University Hospital of Freiburg. Consent to publish was obtained.

\section{Consent for publication}

Not applicable.

\section{Competing interests}

The authors declare that they have no competing interests.

\section{Publisher's Note}

Springer Nature remains neutral with regard to jurisdictional claims in published maps and institutional affiliations.

\section{Author details}

'Department of Radiation Oncology, University Medical Center, Freiburg, Germany. ${ }^{2}$ Department of Gastroenterology, Hepatology, Endocrinology and Infectious Diseases, University Medical Center, Freiburg, Germany.

${ }^{3}$ Department of Radiology, University Medical Center, Freiburg, Germany. 
${ }^{4}$ Department of General and Visceral Surgery, University Medical Center, Freiburg, Germany. ${ }^{5}$ Department of Nuclear Medicine, University Medical Center, Freiburg, Germany. ${ }^{6}$ German Cancer Consortium (DKTK), Partner site Freiburg, Freiburg, Germany. ${ }^{7}$ Faculty of Medicine, University of Freiburg, Freiburg, Germany. ${ }^{8}$ German cancer Research Center (DKFZ), Heidelberg, Germany. ${ }^{9}$ Berta-Ottenstein-Programme, Faculty of Medicine, University of Freiburg, Freiburg, Germany.

\section{Received: 26 February 2017 Accepted: 4 July 2017}

Published online: 12 July 2017

\section{References}

1. Ferlay J, Soerjomataram I, Dikshit R, Eser S, Mathers C, Rebelo M, Parkin DM, Forman D, Bray F. Cancer incidence and mortality worldwide: sources, methods and major patterns in GLOBOCAN 2012. Int J Cancer. 2015;136: E359-86.

2. Bruix J, Sherman M. American Association for the Study of liver D: management of hepatocellular carcinoma: an update. Hepatology. 2011;53: 1020-2.

3. Ma S, Jiao B, Liu X, Yi H, Kong D, Gao L, Zhao G, Yang Y, Liu X. Approach to radiation therapy in hepatocellular carcinoma. Cancer Treat Rev. 2010;36:157-63.

4. Llovet JM, Brú C, Bruix J. Prognosis of hepatocellular carcinoma: the BCLC staging classification. Semin Liver Dis. 1999;19(3):329-38.

5. Bruix J, Gores GJ, Mazzaferro V. Hepatocellular carcinoma: clinical frontiers and perspectives. Gut. 2014;63:844-55.

6. Pan CC, Kavanagh BD, Dawson LA, Li XA, Das SK, Miften M, Ten Haken RK Radiation-associated liver injury. Int J Radiat Oncol Biol Phys. 2010;76: S94-100.

7. Mendez Romero A, Wunderink W, van Os RM, Nowak PJ, Heijmen BJ, Nuyttens JJ, Brandwijk RP, Verhoef C, ljzermans JN, Levendag PC. Quality of life after stereotactic body radiation therapy for primary and metastatic liver tumors. Int J Radiat Oncol Biol Phys. 2008;70:1447-52.

8. Tse RV, Hawkins M, Lockwood G, Kim JJ, Cummings B, Knox J, Sherman M Dawson LA. Phase I study of individualized stereotactic body radiotherapy for hepatocellular carcinoma and intrahepatic cholangiocarcinoma. J Clin Oncol. 2008;26:657-64.

9. Andolino DL, Johnson CS, Maluccio M, Kwo P, Tector AJ, Zook J, Johnstone PA, Cardenes HR. Stereotactic body radiotherapy for primary hepatocellular carcinoma. Int J Radiat Oncol Biol Phys. 2011;81:e447-53.

10. Bujold A, Massey CA, Kim JJ, Brierley J, Cho C, Wong RK, Dinniwell RE, Kassam Z, Ringash J, Cummings B, et al. Sequential phase I and II trials of stereotactic body radiotherapy for locally advanced hepatocellular carcinoma. J Clin Oncol. 2013;31:1631-9.

11. Sterzing F, Brunner TB, Ernst I, Baus WW, Greve B, Herfarth K, Guckenberge M. Stereotactic body radiotherapy for liver tumors: principles and practical guidelines of the DEGRO working group on stereotactic radiotherapy. Strahlenther Onkol. 2014;190:872-81.

12. Wahl DRSM, Tao Y, Pollom EL, Caoili EM, Lawrence TS, Schipper MJ, Feng M Outcomes after stereotactic body radiotherapy or radiofrequency ablation for Hepatocellular carcinoma. J Clin Oncol. 2016 Feb 10;34(5):452-9. 101200/ JCO2015614925 2016

13. Timmerman RD. An overview of hypofractionation and introduction to this issue of seminars in radiation oncology. Semin Radiat Oncol. 2008;18:215-22.

14. Brunner TB, Nestle U, Adebahr S, Gkika E, Wiehle R, Baltas D, Grosu AL. Simultaneous integrated protection : a new concept for high-precision radiation therapy. Strahlenther Onkol. 2016;

15. Fowler JF, Tome WA, Fenwick JD, Mehta MP. A challenge to traditional radiation oncology. Int J Radiat Oncol Biol Phys. 2004;60:1241-56.

16. Pan CC, Kavanagh BD, Dawson LA, Li XA, Das SK, Miften M, Ten Haken RK. Radiation-associated liver injury. Int J Radiat Oncol Biol Phys. 2010;76:94100.

17. Yoo DJ, Kim KM, Jin YJ, Shim JH, Ko GY, Yoon HK, Sung KB, Lee JL, Kang YK, Lim YS, et al. Clinical outcome of 251 patients with extrahepatic metastasis at initial diagnosis of hepatocellular carcinoma: does transarterial chemoembolization improve survival in these patients? J Gastroenterol Hepatol. 2011:26:145-54

18. Qu X-D, Chen C-S, Wang J-H, Yan Z-p, Chen J-m, Gong G-q, Liu Q-X, Luo J-j, Liu L-X, Liu R, Qian S. The efficacy of TACE combined sorafenib in advanced stages hepatocellullar carcinoma. BMC Cancer. 2012;12:1-6.

19. Crane $\mathrm{CH}$, Koay EJ. Solutions that enable ablative radiotherapy for large liver tumors: fractionated dose painting, simultaneous integrated protection, motion management, and computed tomography image guidance. Cancer. 2016;122:1974-86.

20. Que JY, Lin LC, Lin KL, Lin CH, Lin YW, Yang CC. The efficacy of stereotactic body radiation therapy on huge hepatocellular carcinoma unsuitable for other local modalities. Radiat Oncol. 2014;9:120.

21. Huertas A, Baumann AS, Saunier-Kubs F, Salleron J, Oldrini G, Croise-Laurent V, Barraud H, Ayav A, Bronowicki JP, Peiffert D. Stereotactic body radiation therapy as an ablative treatment for inoperable hepatocellular carcinoma. Radiother Oncol. 2015;115:211-6

22. Bibault JE, Dewas S, Vautravers-Dewas C, Hollebecque A, Jarraya $H$, Lacornerie T, Lartigau E, Mirabel X. Stereotactic body radiation therapy for hepatocellular carcinoma: prognostic factors of local control, overall survival, and toxicity. PLoS One. 2013;8:e77472.

23. Mendez Romero A, Wunderink W, Hussain SM, De Pooter JA, Heijmen BJ, Nowak PC, Nuyttens JJ, Brandwijk RP, Verhoef C, ljzermans JN, Levendag PC. Stereotactic body radiation therapy for primary and metastatic liver tumors: a single institution phase i-ii study. Acta Oncol. 2006:45:831-7.

24. Scorsetti M, Comito T, Cozzi L, Clerici E, Tozzi A, Franzese C, Navarria P, Fogliata A, Tomatis S, D'Agostino G, et al. The challenge of inoperable hepatocellular carcinoma (HCC): results of a single-institutional experience on stereotactic body radiation therapy (SBRT). J Cancer Res Clin Oncol. 2015; 141:1301-9.

25. Aitken $\mathrm{KL}$, Hawkins MA. The role of radiotherapy and chemoradiation in the management of primary liver tumours. Clin Oncol (R Coll Radiol). 2014;26: 569-80.

26. Weiner AA, Olsen J, Ma D, Dyk P, DeWees T, Myerson RJ, Parikh P: Stereotactic body radiotherapy for primary hepatic malignancies - Report of a phase I/II institutional study. Radiother Oncol. 2016;121:79-85.

27. Que JY, Lin L-C, Lin K-L, Lin C-H, Lin Y-W, Yang C-C. The efficacy of stereotactic body radiation therapy on huge hepatocellular carcinoma unsuitable for other local modalities. Radiat Oncol. 2014;9:1-8.

28. Min YW, Lee JH, Gwak GY, Paik YH, Lee JH, Rhee PL, Koh KC, Paik SW, Yoo BC, Choi MS. Long-term survival after surgical resection for huge hepatocellular carcinoma: comparison with transarterial chemoembolization after propensity score matching. J Gastroenterol Hepatol. 2014;29:1043-8.

29. Brade AM, Ng S, Brierley J, Kim J Dinniwell R, Ringash J Wong RR, Cho C, Knox J, Dawson LA. Phase 1 trial of Sorafenib and stereotactic body radiation therapy for Hepatocellular carcinoma. Int J Radiat Oncol Biol Phys. 2016;94:580-7.

30. Swaminath A, Knox JJ, Brierley JD, Dinniwell R, Wong R, Kassam Z, Kim J, Coolens C, Brock KK, Dawson LA. Changes in liver volume observed following Sorafenib and liver radiation therapy. Int J Radiat Oncol Biol Phys. 2016:94:729-37.

31. Klein J, Dawson LA, Jiang H, Kim J, Dinniwell R, Brierley J, Wong R, Lockwood G, Ringash J. Prospective longitudinal assessment of quality of life for liver cancer patients treated with stereotactic body radiation therapy. Int J Radiat Oncol Biol Phys. 2015:93:16-25.

32. Feng M, Brunner TB, Ben-Josef E. Stereotactic body radiation therapy for liver cancer: effective therapy with minimal impact on quality of life. Int J Radiat Oncol Biol Phys. 2015;93:26-8.

33. Culleton S, Jiang H, Haddad CR, Kim J, Brierley J, Brade A, Ringash J, Dawson $\mathrm{LA}$. Outcomes following definitive stereotactic body radiotherapy for patients with child-Pugh B or C hepatocellular carcinoma. Radiother Oncol. 2014;111:412-7.

34. Tse RV, Hawkins M, Lockwood G, Kim JJ, Cummings B, Knox J, Sherman M, Dawson LA: Phase I study of individualized stereotactic body radiotherapy for hepatocellular carcinoma and intrahepatic cholangiocarcinoma. J Clin Oncol. 2008:26:657-64.

35. Jang WI, Kim M-S, Bae SH, Cho CK, Yoo HJ, Seo YS, Kang J-K, Kim SY, Lee $\mathrm{DH}$, Han CJ, et al. High-dose stereotactic body radiotherapy correlates increased local control and overall survival in patients with inoperable hepatocellular carcinoma. Radiat Oncol. 2013;8:1-12.

36. Huang W-Y, Jen Y-M, Lee M-S, Chang L-P, Chen C-M, Ko K-H, Lin K-T, Lin JC, Chao H-L, Lin C-S, et al. Stereotactic body radiation therapy in recurrent Hepatocellular carcinoma. Int J Radiat Oncol Biol Phys. 2012;84:355-61.

37. Bae SH, Park HC, Lim DH, Lee JA, Gwak GY, Choi MS, Lee JH, Koh KC, Paik SW, Yoo BC. Salvage treatment with hypofractionated radiotherapy in patients with recurrent small hepatocellular carcinoma. Int J Radiat Oncol Biol Phys. 2012;82:e603-7.

38. Jung J, Yoon SM, Han S, Shim JH, Kim KM, Lim YS, Lee HC, Kim SY, Park JH, $\mathrm{Kim} \mathrm{JH}$. Alpha-fetoprotein normalization as a prognostic surrogate in small 
hepatocellular carcinoma after stereotactic body radiotherapy: a propensity score matching analysis. BMC Cancer. 2015;15:987.

39. Seo YS, Kim MS, Yoo SY, Cho CK, Choi CW, Kim JH, Han CJ, Park SC, Lee BH, Kim YH, Lee DH. Preliminary result of stereotactic body radiotherapy as a local salvage treatment for inoperable hepatocellular carcinoma. J Surg Oncol. 2010;102:209-14.

40. Kwon JH, Bae SH, Kim JY, Choi BO, Jang HS, Jang JW, Choi JY, Yoon SK, Chung KW. Long-term effect of stereotactic body radiation therapy for primary hepatocellular carcinoma ineligible for local ablation therapy or surgical resection. Stereotactic radiotherapy for liver cancer. BMC Cancer. 2010;10:475.

41. Takeda A, Takahashi M, Kunieda E, Takeda T, Sanuki N, Koike Y, Atsukawa K, Ohashi T, Saito H, Shigematsu N: Hypofractionated stereotactic radiotherapy with and without transarterial chemoembolization for small hepatocellular carcinoma not eligible for other ablation therapies: Preliminary results for efficacy and toxicity. Hepatol Res. 2008;38:60-9.

42. Price TR, Perkins SM, Sandrasegaran K, Henderson MA, Maluccio MA, Zook JE, Tector AJ, Vianna RM, Johnstone PA, Cardenes HR. Evaluation of response after stereotactic body radiotherapy for hepatocellular carcinoma. Cancer. 2012;118:3191-8,

43. Kang JK, Kim MS, Cho CK, Yang KM, Yoo HJ, Kim JH, Bae SH, Jung DH, Kim $\mathrm{KB}$, Lee $\mathrm{DH}$, et al. Stereotactic body radiation therapy for inoperable hepatocellular carcinoma as a local salvage treatment after incomplete transarterial chemoembolization. Cancer. 2012;118

44. Su TS, Liang P, Lu HZ, Liang J, Gao YC, Zhou Y, Huang Y, Tang MY, Liang JN. Stereotactic body radiation therapy for small primary or recurrent hepatocellular carcinoma in 132 Chinese patients. J Surg Oncol. 2016;113: $181-7$.

45. Kang J, Nie Q, Du R, Zhang L, Zhang J, Li Q, Li J, Qi W. Stereotactic body radiotherapy combined with transarterial chemoembolization for hepatocellular carcinoma with portal vein tumor thrombosis. Mol Clin Oncol. 2014;2:43-50.

46. Sanuki N, Takeda A, Oku Y, Mizuno T, Aoki Y, Eriguchi T, Iwabuchi S, Kunieda E. Stereotactic body radiotherapy for small hepatocellular carcinoma: a retrospective outcome analysis in 185 patients. Acta Oncol. 2014;53:399-404

47. Kimura T, Aikata H, Takahashi S, Takahashi I, Nishibuchi I, Doi Y, Kenjo M, Murakami Y, Honda Y, Kakizawa H, et al. Stereotactic body radiotherapy for patients with small hepatocellular carcinoma ineligible for resection or ablation therapies. Hepatol Res. 2015;45:378-86.

48. Que J, Kuo HT, Lin LC, Lin KL, Lin CH, Lin YW, Yang CC. Clinical outcomes and prognostic factors of cyberknife stereotactic body radiation therapy for unresectable hepatocellular carcinoma. BMC Cancer. 2016;16:451.

\section{Submit your next manuscript to BioMed Central and we will help you at every step:}

- We accept pre-submission inquiries

- Our selector tool helps you to find the most relevant journal

- We provide round the clock customer support

- Convenient online submission

- Thorough peer review

- Inclusion in PubMed and all major indexing services

- Maximum visibility for your research

Submit your manuscript at www.biomedcentral.com/submit

CBiomed Central 